\title{
Stepping outside the ordinary expectations of schooling: effect of school language on assessment of children's knowledge of mathematics in Ghana
}

\author{
Wilmot, E. M. ${ }^{2}$
}

\section{Abstract}

As a result of barriers erected by language, especially in situations where 'school language' is different from children's mother tongue, there could often be incongruity between children's knowledge of mathematics and teachers' assessment of it. This paper, a case study conducted in Ghana, West Africa, shows how children's 'mother language' can be used to make what is 'invisible' to teachers - children's true knowledge of mathematics - 'visible'. By changing the medium of instruction from the dominant classroom language to the child's mother tongue this study reveals how it takes stepping outside the ordinary to see the weaknesses in teachers' assessment practices.

Keywords medium of instruction, school language, mother tongue, assessing mathematics in early years, mathematics achievement in early years

\section{Introduction}

School culture may not always be congruent with every child's way of learning. However, the incongruence in the ways in which a particular child's culture interacts with the institutional culture may not be always evident to those who manage the school system. The result is that on many occasions the behavior of those children who are adversely affected end up being wrongly interpreted. Mike Rose captures this in a very touching way,

\begin{abstract}
Through all my experiences with people struggling to learn, the one thing that strikes me most is the ease with which we misperceive failed performance. Class and culture erect boundaries that hinder our vision- blind us to the logic of error and the everpresent stirring of language-and encourage the designation of otherness, difference, deficiency (Mike Rose, Lives on the Boundary, p.205).
\end{abstract}

By examining one of the ways in which boundaries that hinder teachers' vision do not become evident, as teachers and students interact, this paper shows how Rose's view is not only true but cuts across cultures and how, in most cases, it takes stepping outside of the ordinary expectations of schooling as an institution, to make the 'invisible' 'visible' (Philips, 1983). This paper focuses on the mathematical ability of two Primary 2 (second grade) students in Ghana to make the familiar strange by revealing how institutions and cultures might come into conflict with one another without being noticed. By comparing the mathematical problem solving behavior of the two students, the paper brings out the negative effects of school language on the way children's mathematical ability are assessed by teachers. It is an offshoot of a broader study conducted in Ghana to investigate Ghanaian children's adding schemes. Though not the direct focus of the

2 Dr. E. M. Wilmot lectures at the Department of Science and Mathematics Education, University of Cape Coast, Ghana. 
Stepping outside the ordinary expectations of schooling: effect of school language on assessment of children's knowledge of mathematics in Ghana Wilmot, E. M.

original $\mathrm{study}^{3}$, something happened with one of the kids, $\mathrm{Elli}^{4}$, a second grader, during the clinical interviews that raises a number of questions about the nature of institutional interactions and their implications for decisions made in schools. In this paper, protocols from the interview with Elli are analyzed and compared with those of another child, Fiifi, to bring out the inconsistencies in the way they were assessed and viewed by their teacher.

Elli's classroom teacher had classified him as a low achieving child (i.e., achieving below the class average). However, the child's performance during the clinical interviews raises a number of questions regarding how children's learning could be affected if school language, the primary school cultural resource, is not available to them and the extent to which their knowledge could be incorrectly assessed. These issues are the foci of this paper.

The paper opens with the background to the study. This is followed by a description of the context of the study. After this, a number of key words have been operationally defined. Next, comes the conceptual framework for characterizing the two children's problem solving behavior followed by a brief account of the procedures used in the study. Data collected in the study is then presented and analyzed. The paper ends with the conclusion where pertinent questions for researchers and practitioners are raised.

\section{Background to the study}

Available literature (Brownell, 1928, 1941; Brownell \& Chazal, 1935; Houlahan \& Ginsburg, 1981; Resnick \& Ford, 1981) indicates that when primary-age children are presented with basic addition combinations they tend to use different strategies. Some children count on their fingers, others use known combinations, while some give immediate answers, which are mostly incorrect, in a manner that indicates that they are guessing. There is evidence that these different strategies are still used even when they have been given common instruction (Ginsburg, 1977b; Adetula, 1990). This is because children are known to assimilate school arithmetic into their existing cognitive structures (Ginsburg, 1977; Skemp, 1987; Hiebert and Lefevre, 1986; RittleJohnson and Siegler, 1998). Therefore, when given addition tasks children attempt to solve them according to the way they organize their experience at that level of development, which they find themselves. Unfortunately, some barriers exist to learning using some of these strategies.

It is therefore necessary for teachers to consider children's strengths and weaknesses when planning instruction no matter how homogeneous they may appear in age so as to help them use what their strategies effectively and develop from there. Thus, instead of designing instruction with the hope that all children in the class would understand, it is necessary, as Brownell (1928) recommended that, "teachers must keep fully informed concerning the stages of development of

${ }^{3}$ The purpose of the original study was to investigate the effect of instruction, tailored to children's level of development, on their strategies for solving addition problems. It was conducted as a two-part study. The first part consisted of individual interviews of the children to assess their levels of development while the second part was a teaching experiment in which the children were taught according to their levels of development as presented in the conceptual framework of this paper.

${ }^{4}$ For confidentiality purposes the actual names of the pupils used in the study have been withheld and instead pseudo names used. 
the pupils by means of continuous study ... of the procedures and processes which the pupils employ in dealing with numbers" (p. 143).

This means that it is necessary for teachers to find out the level of development of their pupils before designing instruction to take care of their differences. This will involve providing them with an opportunity to demonstrate or represent their mental constructs or knowledge externally), use or apply it to situations that require reasoning and creative thinking and communicate that knowledge (NCTM evaluation standard, 1989). That is, by giving them the opportunity to make external their internal representations (Cifarelli, 1998; Pape and Tchoshanov, 2001).

However, a question that arises is, what if a student cannot find the right vocabulary to express himself/herself in a way the best describes his/her thought processes? Should we assume as most teachers do that the fact that he/she cannot explain himself/herself means he/she does not have a conceptual understanding? It is this difficulty that this study sought to unearth in order to bring to the fore the problems associated with they way students are assessed and categorized and in our schools to the point of at times not motivating them to learn.

\title{
The context of the study
}

Ghana is a highly multilingual country with about 60 indigenous languages spoken within its boundaries, none of which is the national language. Rather, English is used as the official language. The history of education in Ghana shows a fluctuating school language policy with a swing between an all-English language policy and transitional bilingual early exit policies in her schools. At the time of this study the language policy for schools in Ghana stipulated that,

\begin{abstract}
In the first three years of primary education, the Ghanaian language prevalent in the local area is to be used as medium of instruction, whilst English is studied as a subject. From Primary four English is to replace Ghanaian language as medium of instruction and the Ghanaian language treated as another subject on the timetable.
\end{abstract}

However, as at the time of the study, this policy was not enforced. As a result, while some schools, especially the rural ones implemented the policy, several others, especially the urban and private schools, had evolved their own policies. An urban primary school in Cape Coast, where this study was conducted was one of the schools where, contrary to the national school language policy, English was used right from the first grade. Teachers in this school only code switched into Fante ${ }^{5}$, the prevalent local language to offer explanations when they suspected that their pupils did not understand.

\section{Definition of terms}

Before discussing the theoretical frame that guided the study, it is essential to define a few terms, which are essential for the analysis of the data in this study.

Counting In this paper, counting is taken to mean the production of a sequence of number words, each accompanied by the production of a unit item. This view is the same as that provided by Steffe, Glasersfeld, Richards and Cobb (1983). This brings us to another term, counting unit item, which I define next.

\footnotetext{
${ }^{5}$ Fante is a dialect of Akan, the most widely spoken indigenous language in Ghana. It is estimated that between 40 and 50\% of Ghanaians are Akans. Akans are from the Ashanti, Central, Brong-Ahafo, and parts of the Eastern Western and Volta regions of Ghana.
} 
Stepping outside the ordinary expectations of schooling: effect of school language on assessment of children's knowledge of mathematics in Ghana Wilmot, E. M.

Counting unit item Bridgman (1961) argues that, "the thing we count was not there before we counted it but we create it as we go along. It is the act of creation that we count"(p.105). Upholding this view, I define a counting unit item as the object that a child is aware of and creates during counting. It could consist of perceptual items, visualized images, finger pointing (head nodding or any signal), vocal production of number words, or the awareness of the conceptual structure of numbers.

Scheme A scheme is here defined as any repeatable action carried out either in the mind (internally) or by the manipulation of objects (externally) while solving a task.

\section{Conceptual Framework}

Steffe, Glasersfeld, Richards and Cobb (1983) have developed a theory involving children's counting units and their adding schemes, which forms the framework used to understand children's level of development in the light of how they solved various addition tasks presented to them. In this section we look at each of these (i.e., children's counting units and their adding schemes) separately and then see how they are connected.

\section{Counting units}

Steffe and her colleagues have shown, form their analysis of the counting behavior of first graders in the context of solving arithmetic tasks that children construct five different types of counting units. These unit items are based on the nature of objects children create and are aware of during counting. The five unit items, in order of sophistication, are perceptual, figural, motor, verbal and abstract units.

To Steffe et al. (1983) a child is called a counter of perceptual unit items if he/she "requires the perceptual component and is unable to count unless a collection of items is actually available (p. 118)." The child's total experience may include the motor act of pointing and verbal production of number words as well. However, there is no awareness on the part of the child that the number words designate the numerosity of the produced perceptual items.

A child is said to be a counter of figural unit items if he/she is able to construct and count figural representations of perceptual, which though they are presented in the context of a task are not perceptually available to him/her at the moment. Such representation need not be an accurate representation of the contextually perceptual items but only result from visual images of the situation present to the child. Also, the child's experience will comprise motor act of pointing and verbal production of number words as well. However, there is neither able to use the number words to designate the numerosity of the visualized items nor use the motor acts for isolating the visualized images into discrete experiential items.

A counter of motor unit items is the child who is aware that he/she could execute his/her pointing or grasping acts independently, substitute them for countable perceptual or figural items and count those motor acts as unit. Steffe and her colleagues maintain that though vocal production of a number word is itself a motor act, it is of a special kind because it is much less apparent to the child than the act of pointing or grasping. To be able to count the verbal acts, the child has to develop a greater awareness than is needed for the other motor experience. It is when a child has 
developed this awareness that number words can be used as substitutes for the previously discussed items that he/she is said to be a counter of verbal unit items.

A child is called a counter of abstract unit items only when he/she is able to go from a number word to the structure, which that number designates. For instance, to solve " $7+5$ ", such a child can use a composite unit represented by the numerosity of " $\&$ " by simply uttering "seven" and then continuing, "eight, nine, ten eleven twelve" (while extending his/her five fingers) to get the answer. According to them, the creation of these unit items constitutes a developmental progression form perceptual units, the least sophisticated, to abstract units, the most sophisticated (in the order in which they have been presented above. However, a counter of a more sophisticated counting unit does not lose the ability to create and count less sophisticated items. The most advanced unit item he/she can create and use therefore classifies a child.

\section{Adding schemes}

The literature on the development of children's adding scheme identifies four schemes (Steffe et al., 1981, 1982, 1983; Eshun, 1985). These four schemes are also identified to be in a similar progression as the counting unit types. In order of sophistication, they are the sensorimotor, preoperational, operational and arithmetic schemes.

According to Steffe et al. (1983), a child with a sensorimotor adding scheme is one who solves additive tasks by globally joining collections of perceptual items. Such a child would use physical objects as aid to form patterns of the addends before joining them to find the sum. By so doing, a sensorimotor scheme child uses counting-all models to solve his/her addition tasks.

The child with the preoperational adding scheme solves additive tasks by joining collections of motor or verbal unit items. Eshun (1985) identified three sub schemes at this developmental level. These are (1) the simple adding scheme involving using perceptual units to count-all as in the case of the sensorimotor scheme, (2) the simple extension scheme in which the child uses any one of perceptual, motor or verbal unit items to model one of the addends and then continues after a pause to count only perceptual items beyond that addend until a collection of the second addend is established and (3) the intuitive extension scheme similar to the simple extension scheme except that motor or verbal units and not perceptual units are used to generate and use for the second addend. Whatever the scheme used, one thing that is clear is that the most advanced unit item children at this developmental level could use is the verbal units.

A child at the operational scheme level of development, apart from using the aforementioned lower level schemes, is also capable of creating and using abstract unit items to construct composite units as he/she solves additive tasks. Such a child can, for instance, use what is referred to as a numerical extension scheme by performing a sequential tacit integration for the first addend and from there, use perceptual, verbal, motor or abstract unit items. Due to the ability to use two composite units to arrive at a third one, he/she can solve missing addend tasks as well as derived fact strategy. However, Eshun (1985) argues that the number facts that can be used by such a child are limited to facts involving numbers less than ten or by adding single digits to ten.

Finally the child at the arithmetic scheme level of development solves addition tasks by using transformed (decomposed) composite units to solve addition tasks. These decomposition strategies involve adding to a decade, where the child interprets an addition task as adding a number to the larger addend to yield the next decade and then increasing the new decade by the 
Stepping outside the ordinary expectations of schooling: effect of school language on assessment of children's knowledge of mathematics in Ghana Wilmot, E. M.

difference or recalling sums using place value. As already mentioned such a child can also use all the aforementioned lower developmental level schemes. Like the counting types, a child at a higher or more sophisticated adding scheme does not lose the ability to use lower level schemes to solve different tasks.

\section{Relationship between counting unit items and adding schemes}

When tasks are presented to children, they organize their thoughts based on what they meanings and understanding they derive from their individual interpretations or assimilation (Skemp, 1987; Eshun, 1985; Ginsburg, 1977). The child's solution path therefore depends on how the assimilation is done and the type of unit items he is capable of creating and using. This then implies that there must be a relationship between a child's counting scheme and his/her adding scheme. From the literature it is clear that a child who uses the sensorimotor scheme only creates and counts perceptual units while the preoperational scheme child counts both motor and verbal unit items. A child classified to be using operational scheme is a counter of abstract units just as the child with the arithmetic scheme. However, as mentioned earlier, the difference between the operational and abstract scheme child is the inability of the former to use number facts with addends larger than ten (Eshun, 1985).

\section{Research design and method}

A week before to the study, the first- and second-grade teachers in the school were asked to prepare a list of their class into three ability groups; above class average performers, just at average and below class average performers in mathematics. In each of the two classes five children were then selected at random from each ability group resulting in 15 first- graders and another 15 from the second grade. After this, visits were paid to the homes of the selected pupils to brief parents about the purpose of the study and obtain permission for their children to participate and enquire about the language of communication at home. Fortunately, no parent turned down the son or daughter's selection to participate in the study.

As already mentioned, the main study was conducted as a teaching experiment (Steffe et al., 1983; Kantowski, 1978). It involved observing the behavior of the participating children and probing their mental processes during individual clinical interviews (Opper, 1977) after which teaching episodes were conducted.

However, this paper focuses only on the first part of the study. It consisted of individual clinical interviews of the 30 selected children by probing each child's counting processes and problem solving behavior using tasks. These interviews were conducted in the school's library, a separate room, distinct from the children's classroom. Each child was given a number of concrete situations, adapted from Steffe et al. (1983) and verbally presented with addition problems related to these situations (see Appendix I). Other tasks, both direct and missing addend nonworded tasks, were presented orally as well as with a function machine. Schematic diagrams of addition tasks simulated using the function machine is shown in Appendix II. During the interviews, each child was given pencil, eraser and a plain sheet as well as a hundred counters to aid the performance of the tasks presented to them. In all cases, children were encouraged after getting an answer to use other strategies they were capable of. Every activity of the interview session was video recorded for analysis later. A child's responses and actions enabled certain inferences to be made about his or her mental processes using the study's framework as a lens. 
The interviews lasted for between twenty and forty minutes per child. In the section that follows, data from the study is both presented and analyzed.

\section{Background of the selected children}

Though 30 children participated in the clinical interviews, this paper focuses on two of the selected second-grade children, Fiifi and Elli, who due to the diverse background and behavior during the study provide useful insights on to how language can affect the way children's mathematical knowledge often get assessed. Fiifi and Elli were of comparable ages, seven years six months and seven years eight months respectively, at the time of the study. Fiifi is a Fante from Cape Coast whose parents were professors at the University of Cape Coast. Elli, on the other hand is an Ewe ${ }^{6}$ whose parents had about six months before the study moved to Cape Coast due to the father's job posting. His father was a clerk at one of the banks in Cape Coast and the mother is a seamstress.

A visit to their houses indicated that Fiifi interacted with his parents mostly in English at home and sometimes in Fante. He was therefore very proficient in both of these languages. Elli, on the other hand, was not very proficient in English or Fante. At home, he interacted with his parents mostly in Ewe. Elli's parents explained that they had started speaking English with him at home because they realized that was the main medium of instruction at his new school. Though Elli had begun to speak the prevalent Ghanaian language of the area and a little bit of English, the media of instruction in her classroom, he could not use them meaningfully in the learning process.

Thus, whereas Fiifi could be considered as having the communicative competence of either English language or Fante because of the effective manner with which he communicated in them, Elli was not. As a result, while Fiifi was very active in class, Elli was most of the time either silent or not participating actively in class. According to their teacher's assessment, Fiifi was performing above the class average while Elli was just an average performer.

\section{Results and Discussion}

\section{Elli and Fiifi's behavior during the interviews}

Data from this study is qualitative in nature and presented in the form of protocols from the interviews so as to bring out the nature interaction at each interview session and illustrate how Elli (E) and Fiifi (F) behaved in their respective sessions with the researcher (R).

\section{Unit items created by Elli}

During the interviews both Elli's counting unit type was identified. The following protocols illustrate this.

In the first protocol, an oral task was presented orally to Elli and he was encouraged to use any preferred strategy.

\footnotetext{
${ }^{6}$ Ewes are about the third dominant ethnic group (constituting about $13 \%$ of the population) in Ghana after the Akans and Moshi-Dagomba (about 16\%). Ewes in Ghana come from the Volta region.
} 
Stepping outside the ordinary expectations of schooling: effect of school language on assessment of children's knowledge of mathematics in Ghana Wilmot, E. M.

Protocol $\mathrm{E}_{1}$

R: What is 7 plus 5 ?

E: (Put up his left fingers while successively uttering) 1, 2, 3, 4, 5. (Pauses for about five seconds. Then utters) "seven" (and continues), 8, 9, 10,11,12 (while extending each of his five fingers simultaneously from the count of eight). It is twelve.

In this protocol Elli was able to perform a motor act, pointing of his fingers, accompanied by the vocal production of number words in order to create his counting items. It is clear from the way he used his fingers that he used motor unit items. However, he was not classified with this because he created and used more advanced unit items as the next protocol shows.

Elli was presented with a wooden board, on which were twelve discs in a row - the first seven covered. This is illustrated below.

\section{Protocol $\mathrm{E}_{2}$}

R: There are seven counters under this cloth (pointing to the cloth). I will let you feel this one (he is allowed to feel the first covered disc). How many counters are on the board altogether?

E: (Silent. He gazes at the cloth for about five seconds and responds) Twelve.

R: Tell me how you did it.

E: I just know.

All efforts to get Elli to explain his solution of this and similar tasks failed. He just would not explain. This happened a lot during the interview with Elli. He was able to answer the oral questions presented to him and which demanded few word answers with ease. However, he remained silent and refused to talk any time he was asked to explain how he got his answers or was given similar questions but which demanded expressing himself in English or Fante in answering. It was therefore hypothesized that language could be the barrier. To test this hypothesis questions were reframed in the child's mother tongue any time he was unable to talk. The protocol below is a translation of his explanation when he was encouraged to offer an explanation of his solution in protocol 2 above in Ewe.

E: (Attempts to touch each of the covered discs in turn but is not allowed to do that beyond the first covered disc). Sub vocally utters 7 while gazing at the cloth (and then continues looking successively at the visible counters) $8,9,10,11,12$. Twelve.

In this way, Elli was able to unite the counting acts "one, two three, . . . seven" into a composite unit through his utterance of "seven" and use the single number word, "seven", to designate its structure and count on from there to "twelve" to get his answer. In line with Steffe et al. (1983)'s argument he was classified with the most advanced unit items he was able to create and use. Elli was therefore classified as a counter of abstract unit items.

\section{Unit items created by Fiifi}

Fiifi, like Elli, was also able to create and use very sophisticated as well as less sophisticated unit items. For instance, Fiifi was able to create motor units as well as abstract units. The following protocols illustrate the first of these.

Protocol $\mathrm{F}_{1}$

R: (Presents two screened and three visible counters to Fiifi). There are some counters under this cloth (point) and there are these uncovered ones. I counted them all and they were 5 altogether. Count back starting at 5 to find how many are under the cloth. 
F: (utters) 5, 4, 3, (while touching the visible counters sequentially) and then continues 2, 1(while clenching his fingers).

R: So how many are under the cloth?

F: (Looks at the clenched fingers and utters) two.

Fiifi, in this protocol used the motor act of clenching his finger accompanied by vocal production of number words " 2,1 " to create his countable items. He was in this way therefore counting motor units similar to what we saw of Elli in Protocol $E_{1}$ except that the nature of the tasks were not the same. The next protocol illustrates how Fiifi created and used abstract unit items.

\section{Protocol $\mathrm{F}_{2}$}

R: What is 5 plus 7 ?

F: (Sits quietly for about 10 seconds and utters) twelve.

R: You are right. How did you find your answer?

F: I counted in my head.

R: Can you tell me how you counted it?

F: $\quad$ I took 7 and added the 5 .

R: Do it for me to see.

F: (utters) 7, (and then continues) 8, 9, 10, 11, 12 (while sequentially pointing on the sheet before him). It's 12.

The fact that he could simply utter the number word "seven" to represent the conceptual structure, which the number designates, and deliberately keep tract of the next counting acts that followed made him to be classified as a counter of abstract unit items.

\section{Elli's Adding Action}

The protocol below shows the most sophisticated adding scheme used by Elli during the interview with him. He was presented with an oral task and was encouraged to use any strategy he liked to arrive at his answer.

Protocol $\mathrm{E}_{3}$

R: Elli, can you tell me in Ewe what 17 plus 15 is?

E: (Pauses for about five seconds and responds) Yes, it is 32

R: That is correct. Now explain, in Ewe, how you got the answer.

E: I know that 15 plus 15 is 30 . So I added two more because of the seventeen.

Elli, in this part of the protocol, used a derived fact strategy to arrive at his answer. This ability to use two composite unites to arrive at a third one, according to the framework is shared by children who use the operational and arithmetic schemes. As already mentioned, Eshun (1985) had asserted that the former scheme's use of this strategy is limited to facts involving numbers less than ten. To test this assertion, Elli was further encouraged to use a different strategy. Below is what he did.

R: Now Elli, I want to learn more from you. Can you use a different strategy to solve this problem? I mean 17 plus 15.

E: (Pauses for about 12 seconds) 15 is 10 and 5 (writes 10 and 5 on the sheet of paper). If I add the 10 to the 17, I will get 27 (writes 27 down). 3 from the 5 will make this (points to the 27 ) 30 and 2 more gives 32 .

It is evident from this solution that Elli was capable of transformed composite units to solve addition problems. He used the method at two levels. First he decomposed 15 into 10 and 5 . Then after adding the ten, he split 5 into 3 and 2 in order to add the 3 to the resultant 27 to get the 
Stepping outside the ordinary expectations of schooling: effect of school language on assessment of children's knowledge of mathematics in Ghana Wilmot, E. M.

next decade before increasing the new decade by 2 . He was therefore classified to be at the arithmetic scheme level.

\section{Fiifi's Adding Action}

Fiifi's most advanced adding scheme during the interviews was the arithmetic scheme. An example of this is presented in the protocol below in which he solves the missing addend task "24 + [ ] = 34 presented using the function machine (see Appendix II for how this machine was used).

\section{Protocol $\mathrm{F}_{3}$}

R: (Gives a card with the numeral "24" on it to Fiifi) Fiifi what is on this card?

F: Twenty-four.

R: Put it in the first "IN" hole.

F: Fiifi complies.

R: Now Fiifi, with your eyes closed, hold this card and I will guide you to put it in the second "IN" hole.

F: (Again Fiifi complies and is helped to put in the second card)

R: Now go to the "OUT" hole and take out what the machine gives us.

F: Fiifi complies and brings out a card bearing the numeral " 34 "

R: What do you think was put in the second "IN" hole while your eyes were closed?

F: (Pauses for about 5 seconds and utters) ten.

R: how did you find out?

F: I took the " 4 " in the " 24 " aside and added the 10 to the 20 left to get 30 . Then I added the " 4 " to it to get 34 . So the answer is 10 .

Thus, Fiifi was able to use a decomposition strategy to solve this and other missing addend tasks (like $13+[$ ] =25). As a result, he was classified as being at the arithmetic scheme level.

However, as shown in protocol $\mathrm{F}_{2}$ above, Fiifi did not only use the arithmetic scheme to solve the entire addition tasks given. He also used other schemas as well. For instance an operational scheme (i.e., the numerical extension scheme) as well as depicted by the aforementioned protocol by counting-on and deliberately keeping tract of his number word utterances for " 5 ", the other addend. This confirms Steffe et al. (1983)'s assertion that children at more advanced level of development are capable of using less sophisticated strategies on some of the tasks given them.

\section{Conclusion}

In one sense, this study provides a useful example for illustrating one of the things that could result from cultural and institutional incongruence, which when not identified early could deny some children the learning opportunity school is supposed to offer. Data from this study indicates that Elli and Fiifi were quite identical in their counting behavior as well as the adding schemes they were capable of using. They could both create and use abstract unit items, the most sophisticated counting units, in counting as evident from protocols $E_{1}$ and $E_{2}$ for Elli and $F_{2}$ for Fiifi. In addition, they could use the scheme, the most advanced adding scheme to solve addition problem (see protocols $\mathrm{E}_{3}$ and $\mathrm{F}_{3}$ ). From these it could be inferred that these two children could solve similar difficulty level tasks. The only difference between the two children had to do with language competence. Whereas, Fiifi, by virtue of his background was competent in both English and Fante, the two languages used for instruction in the school, Elli's background could not 
permit him to achieve the same competence in either of the two languages at the time of the research.

It was this low competency level in the two languages used frequently in classroom instruction and subsequent difficulty he faced expressing his thoughts in class that was the cause of his classification as an average performing child.

From the foregoing, it is clear that Elli's difficulty in participating actively in class and demonstrating his knowledge of mathematics concepts is simply one of language-his mother tongue that he was competent in versus those languages used in school which he was not very competent in. His situation can be summarized in the words of Harriet Romo (2001) when she said,

In the home, the child appeared to be bright, motivated, and a quick learner. In the classroom, she appeared to lack motivation and did not participate in activities. Without an understanding of cultural differences, lack of participation might have been interpreted negatively by the teacher. The teacher's lack of understanding of these cultural differences caused the child to miss important learning opportunities (p.1).

In short, Elli is prevented from participating fully in classroom learning generally and in the learning of mathematics in particular and the demonstration of his conceptual understanding because he is denied access to the most valuable cultural resource, the language of schooling. This was not, however, his choice; the classroom language was chosen not based upon his needs, but upon the cultural and institutional assumptions of those who hold power in the school-the policy makers, administrators within the educational system in Ghana as well as teachers in his school. Elli's language difficulties were not readily evident; she was considered by his teacher as 'well-prepared' to function socially and academically in school, yet this was not the case. Such Cultural misunderstandings if not detected early and worked on could result in lost educational opportunities (Philips, 1983; Taylor, 1991).

In another sense, this study shows how in some cases it takes stepping outside the ordinary expectations of schooling to reveal the incongruence within the school system. Using the child's 'social language' to determine her true knowledge of mathematics concepts, this study disrupted the ordinary school discourse, changing the medium of instruction from the dominant classroom languages to the child's mother tongue. In this way, what was 'invisible' to the teacher-Elli's true knowledge of mathematics - was made 'visible' (Philips, 1983). That is, it took not following the norms of the classroom and using Ewe, Elli's mother tongue instead of code switching between English and Fante (i.e., stepping outside the ordinary expectations of the school), a source of conflict was revealed between the child's knowledge and her teacher's assessment of it.

A number of pertinent issues come out of this paper. First, there is the need for teachers to come to know their students as individuals and be willing to learn about their background. This will help teachers understand the behavior of each child and his/her strengths and weaknesses in order to motivate him/her to succeed in school. Second, instead of misperceiving failed performance, teachers should have high academic expectations for each child and encourage them to give off their best. There is also the need for parents to work together with teachers in the education of their children. Such collaboration will make it impossible for cultures to blind visions to the point of denying some children the chance of learning. Finally, there is the need for researchers, teachers and teacher educators, to collaborate to find the best way to deal with 
Stepping outside the ordinary expectations of schooling: effect of school language on assessment of children's knowledge of mathematics in Ghana Wilmot, E. M.

multiculturalism in schools since school climates where dominant cultures have assumed control have been known only to benefit children of the dominant cultures and marginalize the vast majority from the less dominant cultures.

\section{References}

Adetula, L. O. (1990). Strategies and Skills used by Primary School Children to Solve Simple addition and Subtraction word Problems. ABACUS - Journal for the Mathematical association of Nigeria, 20, 65-76.

Bridgman, P. W. (1961). The Way Things Are. New York: Viking Compass.

Brownell, W. A. (1928). The Development of Children's Number Ideas in the Primary Grades. Supplementary Education Monographs, No. 35. Chicago: University of Chicago Press.

Brownell, W. A. (1941). Arithmetic in Grades I \& II: A Critical Summary of New and Previously Reported Research. Duke University research studies in Education, Durham: Duke University Press No. 6

Brownell, W. A., \& Chazal, C. B. (1935). The Effects of Premature Drill in Third Grade Arithmetic. Journal of Education Research, 29, 17-28.

Cifarelli, V. V. (1998). The development of Mental Representations as a Problem Solving Activity. Journal of Mathematical Behavior, 17(2), 239-264.

Eshun, B. A. (1985). Children's Adding and Subtracting Schemes. Doctoral Thesis presented to the University of Georgia. Athens, Georgia.

Ginsburg, H. P. (1977). Children's Arithmetic: The Learning Process. New York: D. Van Nostrand.

Ginsburg, H. P. (1977b). The Psychology of Arithmetic Thinking. The Journal of children's Mathematical behavior, 1 (4), 1-89.

Hiebert, J., \& Lefevre, P. (1986). Conceptual and Procedural Knowledge in Mathematics: An Introductory Analysis. In Hiebert (Ed.), Conceptual and Procedural Knowledge: The case of Mathematics. Hillsdale, NJ: Lawrence Erlbaum Associates, pp. 1-27.

Houlahan, D. M. \& Ginsburg, H. P. (1981). The Addition Methods of First- and Second- Grade Children. Journal for Research in Mathematics Education12, 95-106.

Kantowski, M. G. (1978). The Teaching Experiment and Soviet Studies of Problem Solving. In L. L. Hartfield (Ed.), Mathematical Problem Solving. Columbus, OH: ERIC Center for Science, Mathematics, and Environmental education.

National Council of Teachers of Mathematics (1989). Evaluation Standard

Opper, S. (1977). Piaget's Clinical Method. The Journal of Children's Mathematical Behavior, 1 (4), 90-107.

Pape, S. J., \& Tchoshanov, M. A. (2001). The role of Representation(s) in Developing Mathematical Understanding. Theory into Practice, 40(2), 118-127.

Philips, S. U. (1983). Invisible Culture: Communicaiton in the Classroom and Community on the Warm Springs Indian Reservation. New York: Longman.

Resnick, L. B. \& ford, W. W. (1981). The Psychology of Mathematics for Instruction. Hillsdale, NJ: Lawrence Earlbaum. 
Rittle-Johnson, B., \& Siegler, R. S. (1998). The Relation between Conceptual and Procedural Knowledge in Mathematics: A Review. In C. Donlad (Ed.), The development of Mathematical Skills (pp. 75-110). East Sussex, UK: Psychology Press.

Romo, H, D. (2001). Celebrating Diversity to Support Student Success. A presentation given at the National Rural Education Association convention in Albuquerque in October 2001. The theme for the convention was "Rural Education: Celebrating Diversity."

Rose, M. (1989). Lives on the Boundary: The Struggles and Achievements of America's Underprepared. New York: Free Press.

Skemp, R. R. (1987). The Psychology of Learning Mathematics (Expanded American Ed.). Hillsdale, NJ: Lawrence Erlbaum.

Steffe, L. P., Firth, D., \& Cobb, P. (1981). On the Nature of Counting activity: Perceptual Unit Items. For The Learning of Mathematics, 2, 13-21.

Steffe, L. P., Glasersfield, E. V., Richards, J., \& Cobb, P. (1983). Children's Counting Types: Philosophy, Theory and Application. New York: Praeger Scientific.

Steffe, L. P., Thompson, D. W., \& Richards, J. (1982). Children's Counting in Arithmetic Problem Solving. In T. P. Carpenter, J. M. Moser, \& T. A. Romberg (Eds.) Addition and Subtraction: A Cognitive Perspective. Hillsdale, NJ: Lawrence Erlbaum.

Taylor, D. (1991). Learning Denied. Portsmouth, NH: Heinemann. 


\section{Appendix I}

\section{Interview questions, selected from those used by Steffe et al. (1983).}

1. (Six counters in a row)

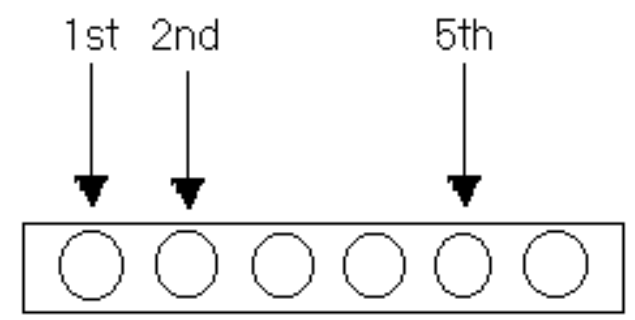

Here are some counters in a row. If we start counting from this end (child's left), this one is the first (point), this one is second (point).

(a) This is fifth (point). Which one is this? (Point to sixth.)

(b) This is fifth (point). Which one is this? (Point to fourth.)

(c) This is fifth (point). Which one is this? (Point to third.)

2. (Cover two counters with cloth.)

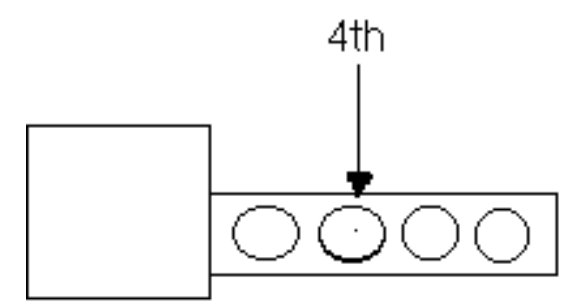

(a) This one fourth (point). How many are under the cloth?

(b) How many are there in all?

3. (12 counters in a row)

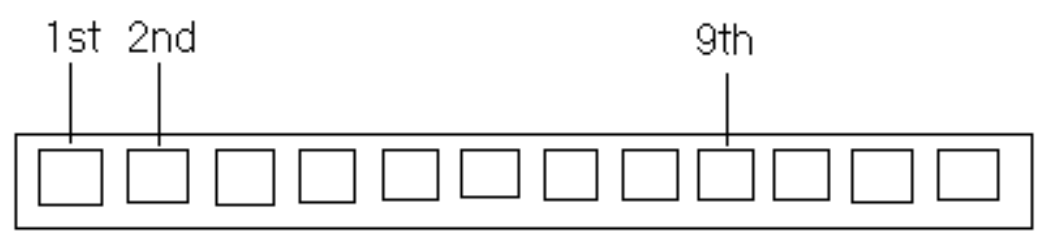

Here are some counters in a row. If we start counting from this end (child's left), this one is first (point), this one is second (point), this one is third (point).

(a) This is ninth (point). Which one is this? (point to tenth).

(b) This is ninth (point). Which one is this? (point to seventh). 
4. (Cover seven counters with cloth)

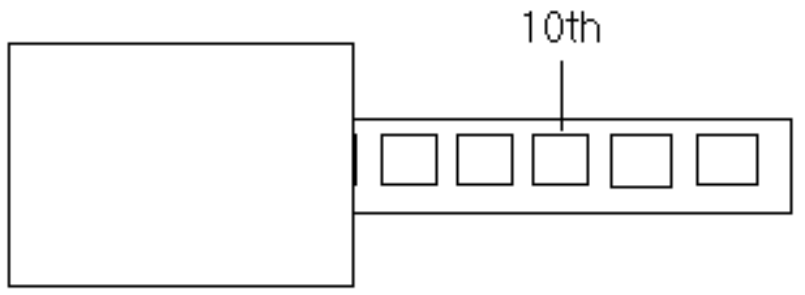

(a) This one is tenth (point). How many are covered?

(b) How many are there in all?

\section{Counting-On}

5. (Two screened checkers and three visible checkers are presented to the child). There are two checkers under the cloth. Count to find how many checkers are there on the card?

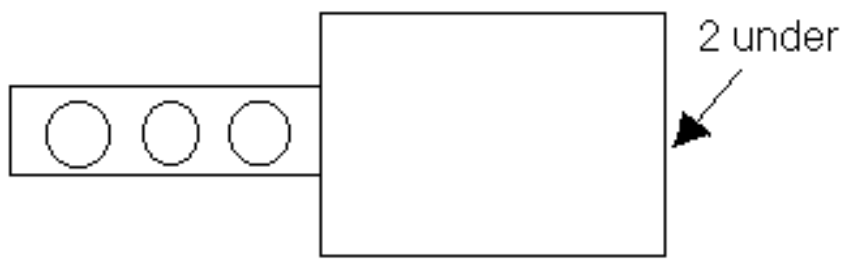

(Two screened checkers and two visible checkers are presented to the child). There are two checkers under the cloth. Count to find how many checkers are there on the card?

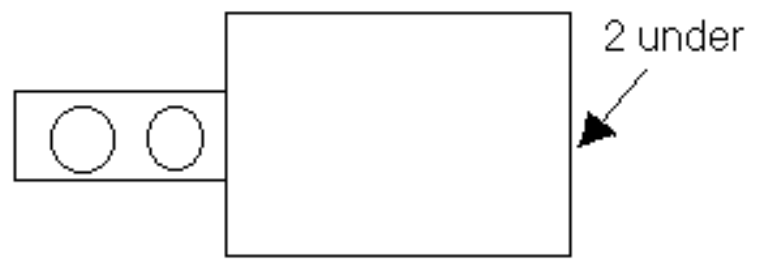

6. Three screened checkers and four visible checkers are presented to the child. There are three checkers under the cloth. Count to find how many checkers are there on the card.

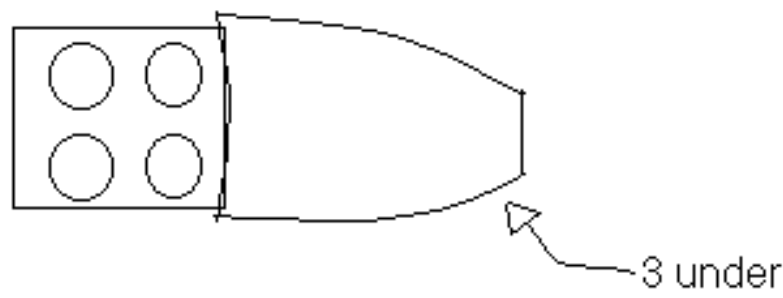


7. (Three screened checkers and five visible checkers are presented to the child). Here are five checkers. There are some more under the cloth. There are eight checkers in all on the card. Count on to find how many checkers are under the cloth.

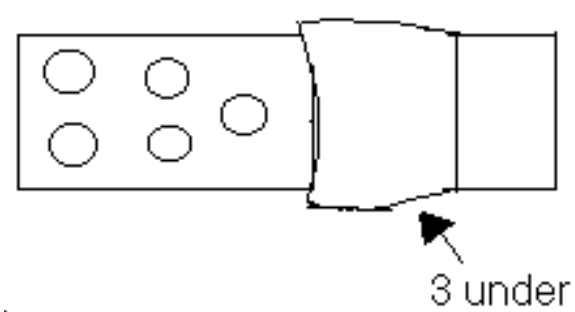

8. (Four checkers, two checkers under each of the two cloths). There are two checkers on the card under this cloth (point) and two more under this cloth (point). Count to find how many are under both cloths.

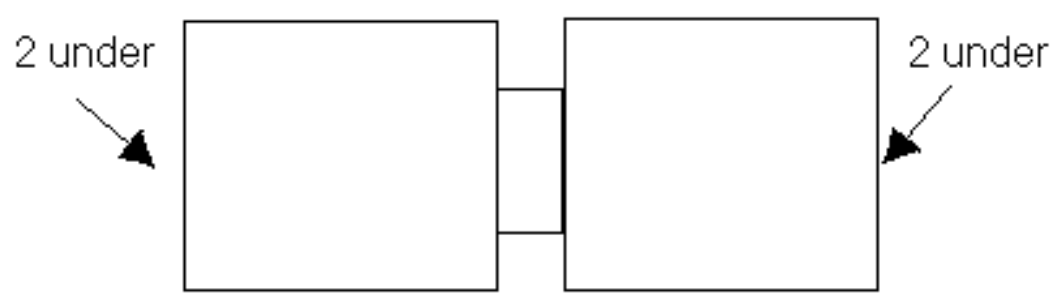

10. (Seven checkers, four under one cloth and three under another cloth are presented to the child). There are four checkers under this cloth (point). There are three checkers under this cloth (point), count to find out how many are under both cloths.

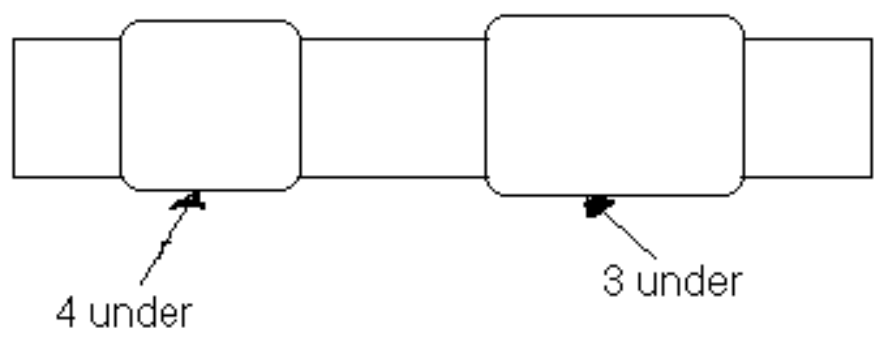

\section{Counting Backward}

11. (A row of five checkers, the first two screened, is presented to the child). There are some checkers under the cloth. I counted them all on the card and there were five. Count back, starting at five, to find how many are under the cloth.

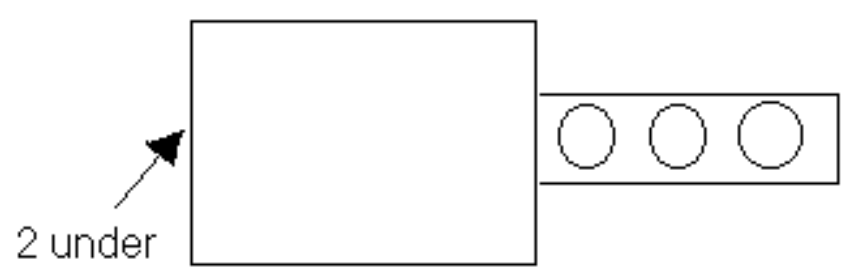


12. (A row of seven checkers, the first four screened, is presented to the child). There are some checkers under the cloth. I counted them all on the card and there are seven. Count back starting at seven, to find how many are under the cloth.

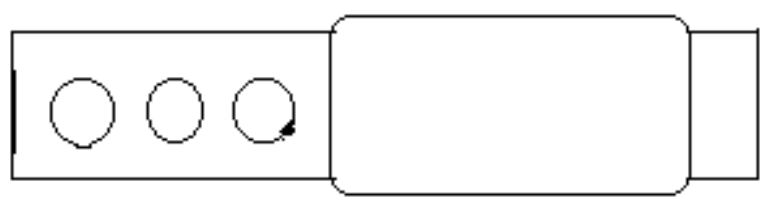

13. (Four checkers, two under the cloth and two under another cloth, are presented to the child). There are four checkers on the card under these cloths (point). There are two checkers under this cloth (point). Count back, starting at four, to find out how many are under this other cloth (point).

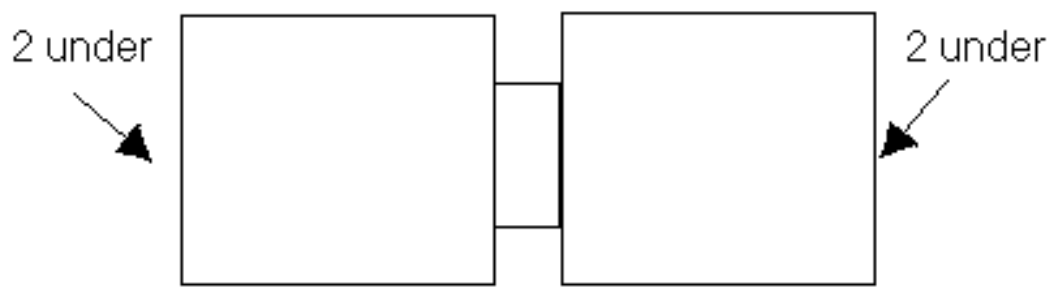

14. (Seven checkers, four under one cloth and three under another cloth are presented to the child). There are seven checkers on the card under these cloths. There are four checkers under this cloth (point). Count back, starting at seven, to find out how many are under this other cloth (point).

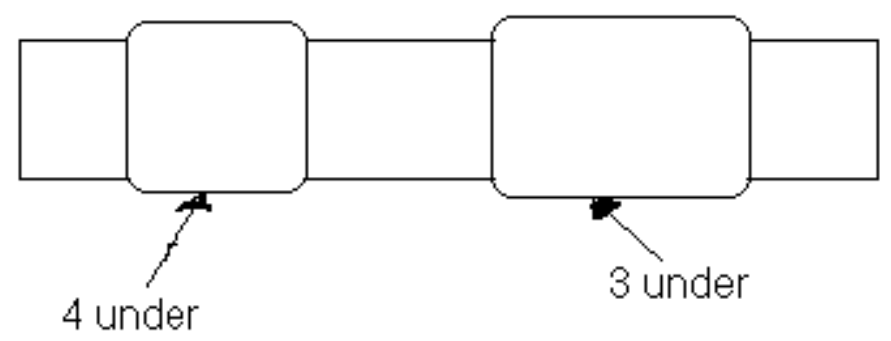


Stepping outside the ordinary expectations of schooling: effect of school language on assessment of children's knowledge of mathematics in Ghana Wilmot, E. M.

\section{Appendix II: Nature of the Function Machine}

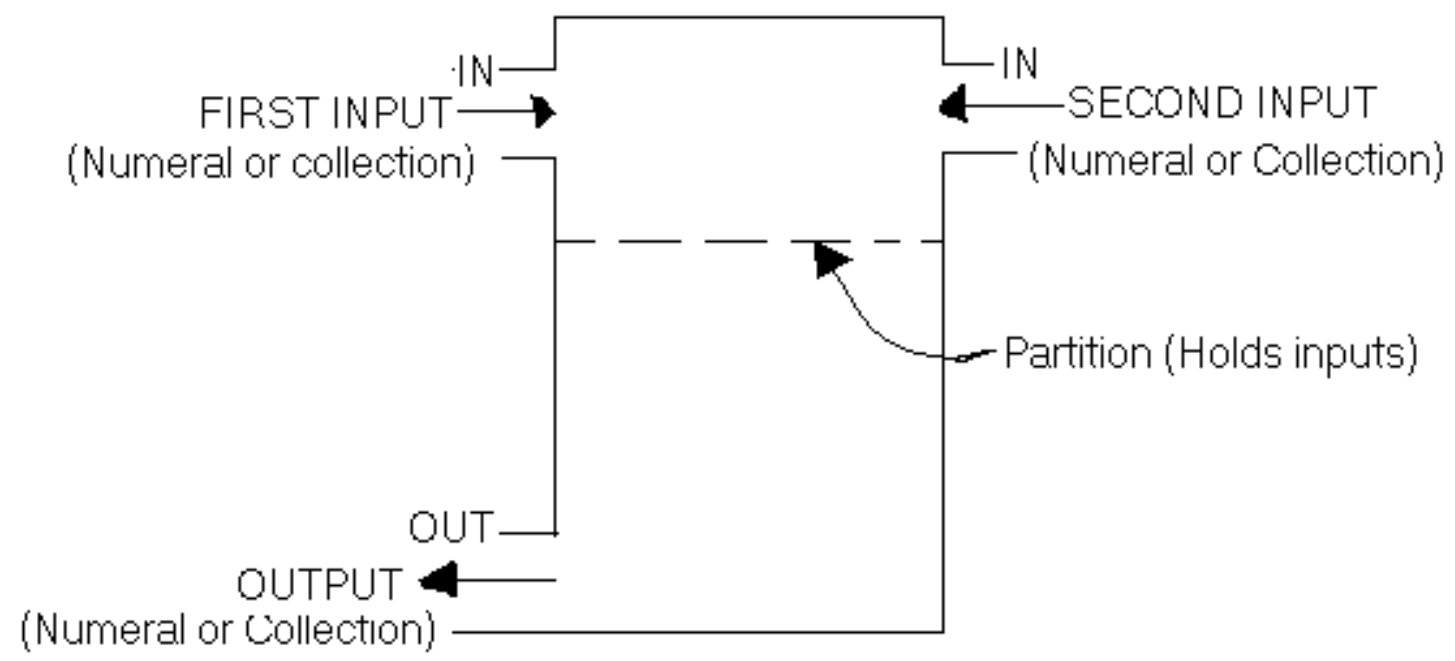

Fig. 1: Function Machine accepting two inputs and yielding an output numeral or collection.

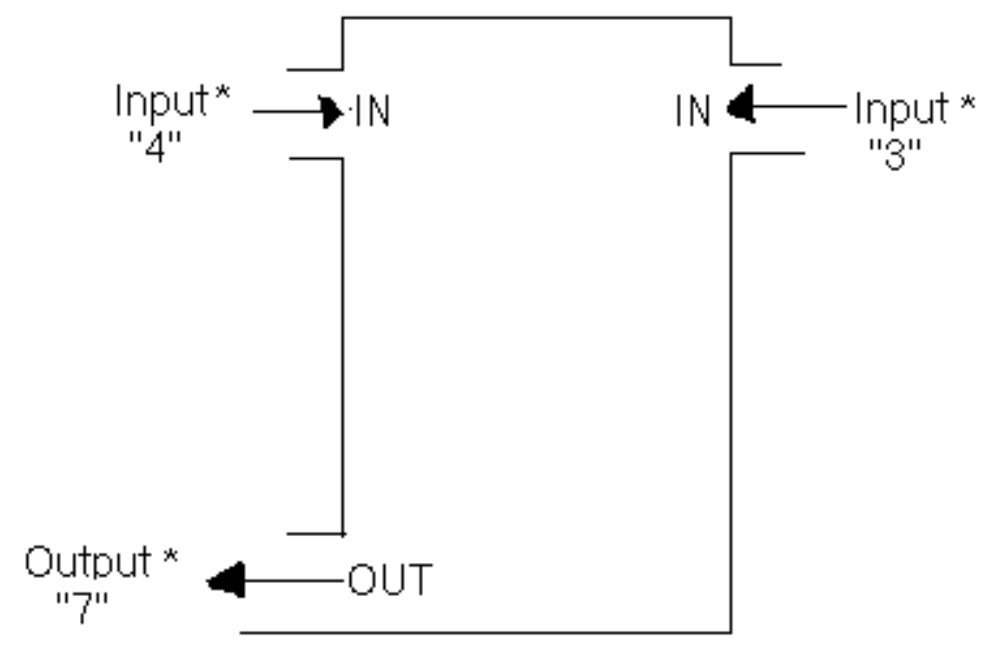

*Card with a Numeral or collection of items drawn on it.

Fig. 2 Direct Addition Simulated Using the Function Machine 